\title{
Modeling tourism using spatial analysis based on social media big data: a review
}

\begin{abstract}
Since an ever-increasing part of the population makes use of social media in their day-to-day lives, social media data has been analyzed in many different disciplines. While there is a great deal of literature on the challenges and difficulties involving specific data analysis methods, there hardly exists research on analyzing the appropriate techniques used to handle different types of data for the purpose of social media analytics. To address this gap, we conducted an extended and structured literature analysis through which we identified challenges addressed and solutions proposed. The literature search revealed that three types of data that were least used for social media analytics that includes Bluetooth, WIFI and mobile roaming data. In contrast, other types of data have received more attention. Based on the results of the literature search, we discuss the most important challenges for researchers and present potential solutions. The findings are used to extend an existing framework on social media analytics. The article provides benefits for researchers and practitioners who wish to collect and analysis social media data.
\end{abstract}

